\title{
A Summary of Cloud Computing Research
}

\author{
Xin Sui ${ }^{1, a}$ and Yi Sui ${ }^{2, b *}$ \\ ${ }^{1}$ College of Humanities and Sciences of Northeast Normal University, Changchun, 130117 \\ ,P.R.China \\ ${ }^{2}$ China Telecom, Beijing Branch Beijing,100124,P.R.China \\ a Suixin1997@126.com; b ericsuiyi@qq.com \\ ${ }^{*}$ The Corresponding author
}

\section{Keywords: Cloud Computing; Information; Sharing Resources; Application}

\begin{abstract}
Cloud computing is a new way of sharing infrastructure. Cloud computing realizes the free circulation of supercomputers through the Internet. The era of cloud computing has arrived. Cloud computing is the product of information technology development, effectively sharing resources. This paper focuses on the development of cloud computing, the definition of cloud computing, the characteristics of cloud computing and the deployment of cloud services. With the integration of big data, cloud computing has good prospects for development and application.
\end{abstract}

\section{The Development of Cloud Computing}

With the rapid development of network communication technology and computer hardware and software technology, the computing mode has undergone different vicissitudes. The original mainframe mode is a centralized computing mode. The request needs to be treated, the processing capacity is limited, and the efficiency is low. With the development of computer miniaturization, computing mode has entered the PC mode computing mode. In 1990s, with the rapid development of network technology, the computing mode entered the network computing mode. Through the network communication equipment, the computer terminals distributed in different regions can be linked together, and they can communicate with each other, share resources and complete different tasks. At this time, many network computing models are proposed, including transparent computing, autonomic computing, [1,2] services computing, [3] computing, [4] and cloud computing, etc.

Cloud computing is the integration and development of distributed computing, web services, virtualization technology, utility computing, network computing and other technologies. Cloud computing virtualization of resources and services on the network, special personnel responsible for all the maintenance, management and scheduling of all resources, users do not need to master the internal implementation.

The essence of cloud computing is that users provide cloud computing services to traditional power, water, and gas - like computing services. [5,6] Which can enable users to maximize the use of virtual resources at any place and at any time through the network, and then solve large-scale computing problems. [7]

\section{Definition of Cloud Computing}

The precise definition of cloud computing is not completely unified at home and abroad.

In 2009, NIST announced the initial definition of cloud computing and then released a revised version of the definition in September 2011: cloud computing is a model that can achieve the required resources, such as applications, storage, servers, and networks at any time, conveniently and on demand, from the shared pool of distributable computing resources. And service. Resources can be quickly supplied and released, so that the workload of management and the intervention of service providers are minimized. [8]

The definition of IBM for cloud computing is a mass of computers connected together to form a large pool of resources, and through virtual technology, the application and network resources are provided through the Internet to supply the user's technical model. 
Wikipedia's definition of cloud computing is that cloud computing is a computing model that is transformed into a service by the network. Users do not need to understand the principles and resources provided by these services. Even without the ability to operate the devices, they can still operate on the interface and connect to the data center through the network, and then complete their work.

The Chinese computer network is defined as cloud computing: the essence of cloud computing is an Internet based supercomputing mode. In remote data centers, a large number of computers and servers are connected to form a cloud.

Users can connect to the cloud through the client of personal computers, mobile phones and other devices, and effectively use resources to complete the calculation according to the requirements of users. Cloud computing allows users to experience super computing power, which can reach more than trillions of times per second.

As early as 2002, Professor Liu Peng pioneered the "computing pool" model, which was confirmed by the "cloud computing" that began in 2007. The "anti-spam grid", which was proposed in 2003, was also confirmed by the "cloud security" that was popular after five years.

In 2003, the anti-spam grid proposed by Professor Liu Peng, through the combination of large data and artificial intelligence, accurately filtered the global spam, and was rated as an outstanding grid project by the IEEE Cluster 2003 International Conference. This technology has been widely used in the world to solve the problem of spam that has plagued mankind for a long time, and has helped China to become a country that can effectively filter spam in the world.

\section{The Basic Features Cloud Computing}

Use on Demand. Cloud users can access cloud based IT resources, and cloud is a huge resource pool. "On demand" refers to the automation of access to IT resources without the involvement of cloud providers or cloud users.

Resource pool. Cloud providers put large amounts of IT resources together (providing services for cloud users) and dynamically allocate resources according to user needs. [9]

Measurability. The cloud platform has the ability to make IT resources record, so it can be easily measured. According to the recorded data, the cloud providers charge corresponding fees for the actual user access time and the specific type.

Elasticity. Flexibility is an effective way to reduce investment cost and use cost, which is generally considered to be the core reason for adopting cloud computing. Users can purchase and use services according to actual needs, and can also revoke or delete resources according to requirements.

Recoverability. Recoverability is achieved by distributing redundant IT resources on multiple physical locations. Then the fault transfer is realized. Distributing redundant IT resources on different physical locations of the same cloud, or redundant IT resources across multiple clouds, can increase the reliability of cloud users. [10]

Virtualization. Virtualization technology makes the server virtual as a virtual machine that can be allocated for performance, and then deploys, manages and regulates the unity of machines in a large scale cluster. When the physical machine load is overloaded, it can move other physical machines from one physical machine to realize load balancing.

\section{Cloud Service Deployment}

The cloud deployment model represents a specific type of cloud environment, and Cloud Computing mainly has four different deployments.

Private Cloud. The private cloud is constructed for the use of an organization alone, only for internal staff or branches of the organization. The private cloud may be physically within the scope of the organization, but all the resources on it are considered to be "cloud based" if it allows cloud users to access remotely.

Because the enterprise has its own infrastructure, it can make corresponding deployment on this infrastructure. It can manage and control data, service quality and security most effectively. 
Private cloud may be managed outside the enterprise or inside the enterprise, either by service providers or by internal IT administrators. In any case, the architecture only serves the enterprise. Enterprises with private cloud must build, purchase and manage cloud environments, which will cost more in the early stage.

There are two ways of deploying private cloud: hosting private cloud and building private cloud.

Public Cloud. Public cloud refers to a cloud environment that can be accessed by third parties, which can be shared by multiple users. Cloud providers are responsible for creating, managing, controlling, and maintaining public clouds.

The advantage of public cloud is that users do not have to invest and build accordingly, and the relevant data, procedures and services are stored in public cloud. It is precisely because the user's data is not stored in their data center, there is a certain degree of security risk.

Hybrid Cloud. Hybrid cloud integrates private cloud and public cloud. The security of private cloud is much higher than that of public cloud, but the computing resources of private cloud are far less than that of public cloud. Hybrid cloud solves this problem perfectly. Hybrid cloud generally refers to the combination of two or more public and private clouds. [11]

Hybrid cloud saves important data in the local data center, which fully reflects the security and reliability of private cloud. At the same time, hybrid cloud can make full use of the resources of public cloud, and accomplish work more efficiently and efficiently. Hybrid cloud has their advantages compared to private cloud and public cloud, and they are more perfect than them.

\section{Acknowledgements}

2018 Jilin Province Education Department "Thirteen-Five" Science and Technology Project "Enterprise Private Cloud Construction Design and Research ", Project No.: JJKH20181306KJ.

\section{Conclusion}

Cloud computing is considered to be the third revolution of the IT industry, and it is the most anticipated new technological revolution in the coming years. Cloud computing has bright prospects for development, and will have great benefits in education, business, communications and other industries. Cloud computing is the main force of the new generation of information industry, which may lead to changes in the business mode of the information industry. In order to accelerate the development of cloud computing industry in China, the Chinese government has selected some cities as a pilot of cloud computing services, and then developed corresponding cloud computing projects.

\section{References}

[1] Murch R.Autonomic Computing.USA:IBM Press, 2004.

[2] Kephart JO, Chess DM. The vision of autonomic computing. Computer, 2003,36 (1): Computer

[3] Papazoglou MP. Service-oriented computing: Concepts, characteristics and directions//Proceedings of the Fourth International

[4] Berman F, Fox G, and Hey AJG. Grid Computing: Making the Making, Fox, 2003.

[5] Armbrust M, Fox A, Grith R, et al. Above the clouds: A.

[6] Vaquero L. Rodero-Marino L, Caceres J, et al. A break in the.

[7] Liu Mengni, Liu Yuzhi, Ma Dong Hao. Application and security overview of cloud computing [J]. network security technology and applications, 2018 (05): 44+54.

[8] Wang Yu. Analysis of computer network cloud computing [J]. China's strategic emerging industries, 2018 (20): 116.

[9] Peng Haoyou, Fu Cuiyu, Yao Jian, Yang Dengpan. Overview of cloud computing [J]. Fujian computer, 2018,34 (01): 1-2+13.

[10] Jia Junting. Cloud computing overview and cloud computing applications in the communications industry [D]. Inner Mongolia University, 2014. 
[11] Yuan Lijuan, Wang Tao. Overview of cloud computing and its current development in China [J]. intelligence, 2011 (07): 80. 